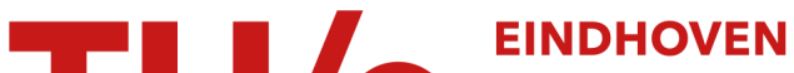 \\ UNIVERSITY OF \\ TECHNOLOGY
}

\section{Droplet behavior in the presence of insoluble surfactants}

Citation for published version (APA):

Stegeman, Y. W., Vosse, van de, F. N., \& Meijer, H. E. H. (2004). Droplet behavior in the presence of insoluble surfactants. Physics of Fluids, 16(8), 2785-2796. https://doi.org/10.1063/1.1756168

DOI:

10.1063/1.1756168

Document status and date:

Published: 01/01/2004

Document Version:

Publisher's PDF, also known as Version of Record (includes final page, issue and volume numbers)

Please check the document version of this publication:

- A submitted manuscript is the version of the article upon submission and before peer-review. There can be important differences between the submitted version and the official published version of record. People interested in the research are advised to contact the author for the final version of the publication, or visit the $\mathrm{DOI}$ to the publisher's website.

- The final author version and the galley proof are versions of the publication after peer review.

- The final published version features the final layout of the paper including the volume, issue and page numbers.

Link to publication

\section{General rights}

Copyright and moral rights for the publications made accessible in the public portal are retained by the authors and/or other copyright owners and it is a condition of accessing publications that users recognise and abide by the legal requirements associated with these rights.

- Users may download and print one copy of any publication from the public portal for the purpose of private study or research.

- You may not further distribute the material or use it for any profit-making activity or commercial gain

- You may freely distribute the URL identifying the publication in the public portal.

If the publication is distributed under the terms of Article $25 \mathrm{fa}$ of the Dutch Copyright Act, indicated by the "Taverne" license above, please follow below link for the End User Agreement:

www.tue.nl/taverne

Take down policy

If you believe that this document breaches copyright please contact us at:

openaccess@tue.nl

providing details and we will investigate your claim. 


\title{
Droplet behavior in the presence of insoluble surfactants
}

\author{
Y. W. Kruijt-Stegeman, ${ }^{\text {a) }}$ F. N. van de Vosse, and H. E. H. Meijer \\ Section Materials Technology, Faculty of Mechanical Engineering, Eindhoven University of Technology, \\ P.O. Box 513, $5600 \mathrm{MB}$ Eindhoven, The Netherlands
}

(Received 15 August 2003; accepted 6 April 2004; published online 9 June 2004)

\begin{abstract}
The time-dependent behavior of droplets in the presence of insoluble surfactants, i.e., droplet elongation in supercritical flow (capillary number $\mathrm{Ca}=0.1$ ) and droplet breakup in a quiescent matrix, is studied using a finite element method. The interfacial tension coefficient $\sigma$ as a function of the surfactant concentration $\Gamma$ is described using the Langmuir equation of state, $\sigma=\sigma_{0}$ $+R T \Gamma_{\infty} \ln \left(1-\Gamma / \Gamma_{\infty}\right)$. For droplets in an equal viscosity system, the influence of parameters $\Gamma, \Gamma_{\infty}$, and the Péclet number (ratio between surfactant convection and diffusion rate) on the elongation behavior has been investigated, whereas droplet breakup is considered for various values of the Péclet number for trace concentrations $\Gamma \ll \Gamma_{\infty}$ of an insoluble surfactant. Depending on the surfactant used, a surfactant covered droplet in supercritical flow may deform more than or less than a clean droplet, as is the case in subcritical flow. Two processes compete: surfactant accumulation near the tips due to convection and overall surfactant dilution due to an increase of interfacial area. Nevertheless, the main effect of surfactants on dispersive mixing is due to the fact that upon breakup, the daughter droplets have a different interfacial tension coefficient. Especially in time-dependent processes, this may have a huge impact on the final droplet distribution. (C) 2004 American Institute of Physics. [DOI: 10.1063/1.1756168]
\end{abstract}

\section{INTRODUCTION}

Many industrial blends contain interfacially active materials, so-called surfactants. Sometimes, surfactants are introduced unwillingly, but since surfactants reduce coalescence, they are often added on purpose to stabilize the morphology. In this study, the influence of insoluble surfactants on timedependent droplet deformation and breakup will be determined using a computational method. ${ }^{1}$ This will be done for an equal viscosity system at different Péclet numbers, i.e., for different ratios between convection and diffusion rate of the surfactant at the interface. Also, the effect of the different parameters describing the relation between surfactant concentration and interfacial tension coefficient will be investigated.

Milliken and Leal ${ }^{2}$ observed a new mode of breakup when they performed experiments to determine the influence of viscoelasticity on droplet deformation using aqueous solutions of polymers. As it became clear a few years later, ${ }^{3,4}$ this new mode of breakup was actually due to the influence of interfacially active materials. These surfactants reduce the interfacial tension coefficient, and may introduce Marangoni stresses if the surfactant concentration gradient along the interface is large. The mode of breakup observed by Milliken and $\mathrm{Leal}^{2}$ has been observed before by, e.g., Bartok and Mason. ${ }^{5}$ At low capillary number, the droplets become pointed, and a stream of very small droplets is ruptured from the ends. The conditions for this type of breakup, which is called tip streaming, were investigated by de Bruijn. ${ }^{6}$ It appears to occur only for low viscosity ratio droplets at low to

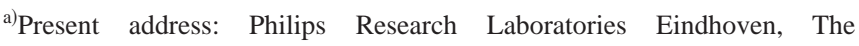
Netherlands. very low concentrations of surfactant. If these conditions do not apply, the type of breakup of surfactant covered droplets resembled the breakup types encountered in clean systems, although breakup times and number of daughter droplets may vary. In subcritical flow, the deformation of surfactant covered droplets may be both higher or lower than the deformation of clean droplets. In general, internal circulation sweeps the surfactant to the tips of the droplet. If the diffusion over the interface and from/to the bulk is slow compared to this convection, the surfactant concentration $\Gamma$ at the droplet tips increases rapidly. The interfacial tension coefficient decreases locally and causes an increased deformation. The gradient in the interfacial tension coefficient introduces Marangoni stresses, which try to force the surfactant molecules away from the tips. Since surfactant molecules occupy space, there is an upper bound $\Gamma_{\infty}$ to the concentration which can be achieved. ${ }^{7,8}$ If this upper bound is approached, the Marangoni stresses become very large and prevent internal circulation. Therefore, a low viscosity ratio droplet will behave as if its viscosity ratio was much larger. ${ }^{4,9-11}$ Whether the upper bound $\Gamma_{\infty}$ is reached, depends on the surfactant coverage $\Gamma / \Gamma_{\infty}$ and the Péclet number Pe, which is the ratio between convection and diffusion along the droplet interface. ${ }^{12}$ For low surfactant coverage, the upper bound is not reached by far, and the droplet deformation will be higher than in the case of a clean droplet. For high surfactant coverage, the relation between the interfacial tension coefficient and the surfactant concentration becomes very strong. In such a case where the Marangoni stresses are large, the droplet deformation becomes dilution dominated and the droplet deforms less than an equivalent clean droplet. ${ }^{10,13-16}$ For higher viscosity ratio droplets, the convection is small 


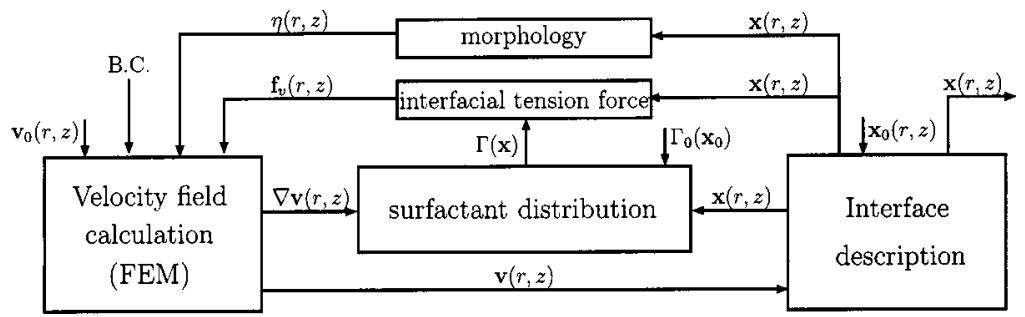

FIG. 1. Schematic representation of the numerical method in case of surfactants.

compared to the diffusion (low Pe) and the surfactant is more uniformly distributed. ${ }^{8}$ Therefore, the effect of surfactant is smaller for higher viscosity ratios. ${ }^{4,9-11,17,18}$

In contrast to previous studies, where the focus was mainly on the determination of the critical capillary number and the behavior of droplets in subcritical flows (steady droplet shape and tip streaming), this study focuses on the time-dependent deformation of droplets in a supercritical elongational flow $\left(\mathrm{Ca}=1.1 \mathrm{Ca}_{c}\right)$. Droplet deformation and breakup as a function of the Péclet number is determined with a finite element method. The relation between the surfactant concentration and the interfacial tension coefficient is described by the Langmuir interfacial equation of state, using various values for the strength of this relation and for the surfactant coverage. Besides the droplet deformation in super critical flow, the influence of surfactant on the breakup of moderately and highly elongated droplets in a quiescent matrix is studied for low surfactant coverage $\left(\Gamma_{r} \ll \Gamma_{\infty}\right)$. For simplicity reasons, only equal viscosity ratio systems are considered. The surfactant is assumed to be insoluble, which is a good approximation if the bulk concentration is dilute ${ }^{12}$ or if the migration to and from the bulk is slow. ${ }^{18}$ In fact, the study by Milliken and $\mathrm{Leal}^{9}$ shows that in almost all cases, the droplet deformation and breakup in the presence of soluble surfactants fall between the results for insoluble surfactant and those for a clean interface.

\section{COMPUTATIONAL METHOD}

In this study, a computational method will be used to determine the influence of insoluble surfactants on the timedependent droplet behavior, i.e., the droplet deformation in supercritical flows and breakup of droplets in a quiescent matrix. In contrast to other studies on droplet deformation, our method is based on a finite element method instead of a boundary integral method. The finite element method has the advantage that it easily can be extended to non-Newtonian fluids, ${ }^{1}$ which is nontrivial for boundary element methods. In this study, however, we only consider equal viscosity Newtonian systems. The numerical method essentially consists of three parts: the description of the interface, the determination of the surfactant distribution, and the computation of the velocity field, see Fig. 1.

The interface is described by a number of marker points, which are connected by linear elements (edges). This interface mesh may be refined and coarsened locally to obtain maximum accuracy at minimum computational cost. On this moving mesh, a convection diffusion equation is solved to obtain the concentration of surfactant in each marker point. Depending on the droplet shape (morphology) and this surfactant distribution, the interfacial tension forces are calculated. Together with the boundary conditions, they will form the input for the velocity field computation. The velocity field is computed on a fixed grid by means of a finite element method. In principle, any finite element method which is able to give approximate solutions of the Stokes equations can be used. At the end of each time step, the marker points are moved according to the computed velocity field (tracking) and the interfacial tension forces are updated.

If we assume inertia to be negligible, droplet deformation is governed by the stationary Stokes equations:

$$
\begin{aligned}
& \nabla p-\nabla \cdot(\eta 2 \mathbf{D})-\mathbf{f}_{v}=\mathbf{0}, \\
& \nabla \cdot \mathbf{v}=0,
\end{aligned}
$$

where $p$ denotes the pressure, $\mathbf{v}$ the velocity and $\eta$ the viscosity. The rate of deformation tensor $\mathbf{D}$ is given by $\frac{1}{2}[\nabla \mathbf{v}$ $\left.+(\nabla \mathbf{v})^{T}\right]$ and $\mathbf{f}_{v}$ denotes a body force. As we consider a two-phase system, a set of additional requirements is introduced on the interface. First of all, the velocity is required to
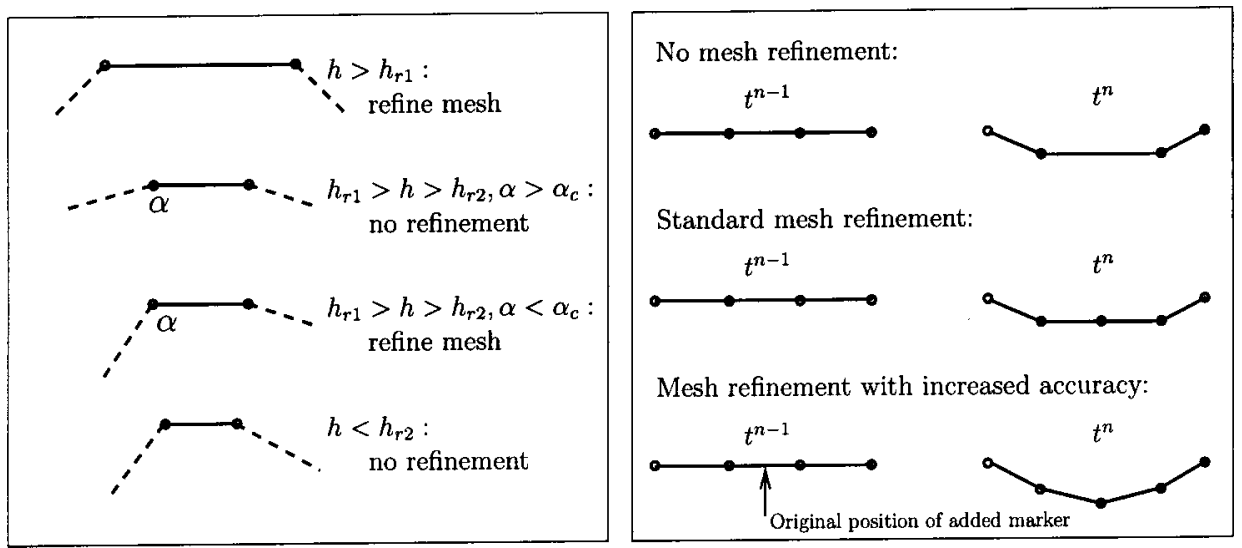

FIG. 2. Mesh refinement is controlled by three parameters: $h_{r 1}, h_{r 2}$, and $\alpha_{c}$ (left). The accuracy of the interface position can be increased by "adding" the new marker in the previous time step and tracking it to its current position (right). 

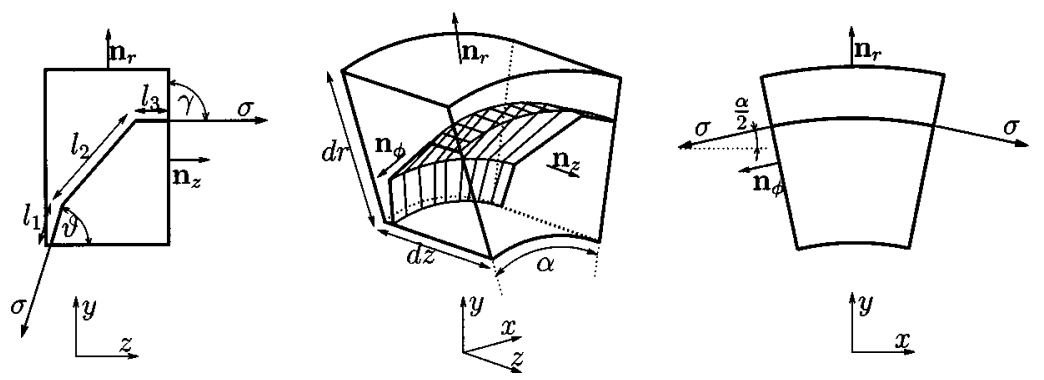

FIG. 3. Interfacial tension on the planes of the segment of the finite element. Using the angles $\alpha$, $\vartheta$, and $\gamma$, the interfacial tension forces can be calculated. be continuous on the droplet interface $\left(\mathbf{v}_{d}=\mathbf{v}_{c}\right)$. Moreover, the interfacial tension introduces a jump in the stress over the interface:

$$
\llbracket \boldsymbol{\tau}+\eta 2 \mathbf{D}-p \rrbracket \cdot \mathbf{n}=\left(\frac{\sigma}{R_{1}}+\frac{\sigma}{R_{2}}\right) \mathbf{n}-\nabla_{s} \sigma,
$$

where $\sigma$ is the interfacial tension coefficient, $\mathbf{n}$ the unit vector normal to the interface, and the surface gradient operator $\nabla_{s}$ is defined as $(\mathbf{I}-\mathbf{n n}) \cdot \nabla$. In any two phase system in which there is a finite interfacial tension present, there is a normal stress difference (Laplace pressure). Additionally, the presence of surfactants will often introduce a gradient in the interfacial tension coefficient. Due to this gradient, a tangential Marangoni stress will be present.

At the end of each time step, the marker points which describe the droplet interface are moved with the velocity field according to $\mathbf{x}_{n+1}=\mathbf{x}_{n}+\int_{t_{n}}^{t_{n+1}} \mathbf{v} d t$. The element size has been chosen such that the markers will move over the distance of one element in about ten time steps. The integration is performed using an adaptive fifth-order Runga-Kutta Cash-Karp scheme. ${ }^{19}$ The velocity at the marker position $\mathbf{v}(\mathbf{x})$ is determined by second order interpolation in space. To obtain the velocity field at intermediate time levels, $\mathbf{v}(\mathbf{x}, t)$, linear interpolation is used between $\mathbf{v}\left(\mathbf{x}, t^{n}\right)$ and $\mathbf{v}\left(\mathbf{x}, t^{n+1}\right)$. Symmetry conditions are imposed by ensuring that the edges adjacent to the $r$-and $z$-axes are perpendicular to these axes. Due to the inhomogeneous velocity field, the distance between some markers will increase in time, whereas the distance between other markers will decrease. Therefore, mesh refinement and coarsening is necessary if the mesh is to remain an accurate description of the interface, while keeping the computational costs low. This mesh refinement is controlled by the length of the edges and the angle between them, see Galaktionov et al. ${ }^{20}$ The mesh is always refined if the edge length $h$ exceeds a critical value $h_{r 1}$. If $h>h_{r 2}$ while at least one of the angles with the neighboring edges is smaller than $\alpha_{c}$ the mesh is also refined, see the left part of Fig. 2. Typically $h_{r 1}$ is chosen to be one-third of the mean element size, while $h_{r 2}=h_{r 1} / 2$ and $\alpha_{c}=160^{\circ}$. The actual refinement consist of splitting the edge into two edges, by adding an extra marker point and updating the topology. To increase the accuracy, the marker is "added" at a previous time step and the already calculated velocity field is used to track it to its current position, see the right part of Fig. 2. Mesh coarsening is needed to remove the marker points which are swept towards the $r$-axis at the tip of the droplet. If the distance between the marker points becomes too small, small errors in the position due to numerical instability can cause large errors in the interfacial tension forces, since these are based on the direction of the edges between the marker points. Mesh coarsening is performed by a procedure similar to mesh refinement $\left(h_{c 1}=h_{r 1} / 3, h_{c 2}=h_{r 1} / 6\right)$.

The interfacial tension is incorporated into the finite element code as a volume force. ${ }^{21}$ This volume force is computed as the total force exerted on an element divided by the volume of this element. In order to show how the volume force is calculated, a small segment of the cylindrical volume element is considered in a Cartesian coordinate system. The droplet interface can be considered to be a membrane. If one cuts this membrane at the intersections with the segment, one has to exert forces on the edges to keep the membrane from collapsing. The position and direction of these forces are given in Fig. 3. For simplicity, we will first consider the case where the interfacial tension coefficient is constant (no Marangoni stresses). The volume force on the total element $(\Sigma \alpha$ $=2 \pi$ ) is given by

$$
\begin{aligned}
\mathbf{f}_{v}= & \frac{2 \sigma}{d z\left(2 d r R_{b}+d r^{2}\right)}\left[\sin (\gamma) r-\sin (\vartheta) R_{b}-d l\right] \mathbf{e}_{r} \\
& +\frac{2 \sigma}{d z\left(2 d r R_{b}+d r^{2}\right)}\left[\cos (\gamma) r-\cos (\vartheta) R_{b}\right] \mathbf{e}_{z},
\end{aligned}
$$

where $R_{b}$ is the radius at the bottom of the segment, $r$ gives the position of the intersection of the interface with plane with normal $\mathbf{n}_{z}$ and $d l$ is the length of the intersection of the interface with the plane with normal $\mathbf{n}_{\phi}: d l=l_{1}+l_{2}+l_{3}$. In a similar manner, the volume forces on all the other elements can be calculated. The volume force in the nodal points is the mean value of the volume forces calculated for the adjacent

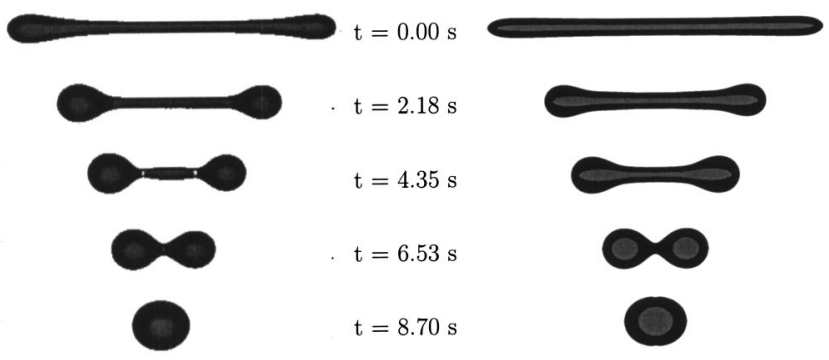

FIG. 4. Relaxation of an elongated droplet in a quiescent matrix. Experimental (left) and numerical (right) results: $\eta_{d}=5.2 \mathrm{~Pa} \cdot \mathrm{s}, \eta_{m}=1.0 \mathrm{~Pa} \cdot \mathrm{s}$, $\sigma=4 \mathrm{mN} / \mathrm{m}, R=0.33 \mathrm{~mm}, L_{0}=6.2 R$. 

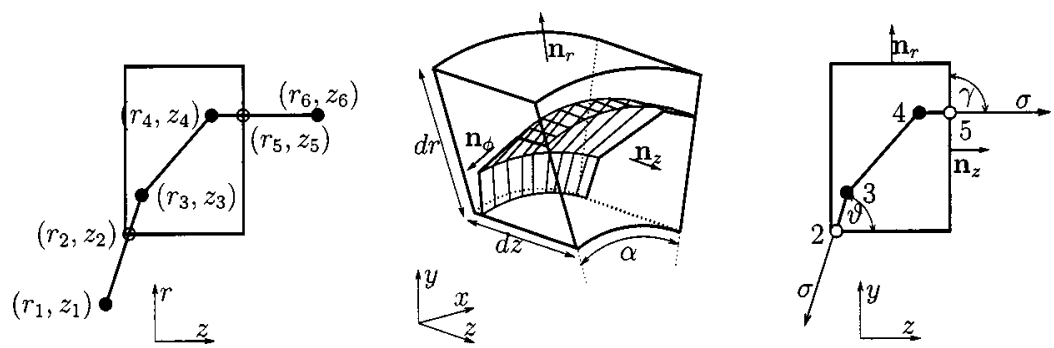

FIG. 5. If surfactants are present, the interfacial tension forces can be calculated using a position dependent interfacial tension coefficient $\sigma(r, z)$. elements. Due to axisymmetry, the volume forces in the $r$-direction of the nodal points on the symmetry axis will be zero.

To prove the validity of the procedure, the interfacial tension driven relaxation of a clean elongated Newtonian droplet in a quiescent matrix has been compared to experimental data. ${ }^{1}$ Figure 4 shows the relaxation of an extended droplet. The experimental data were obtained with a polybutadiene droplet $\left(\eta_{d}=5.2 \mathrm{~Pa} \cdot \mathrm{s}\right)$ of $0.33 \mathrm{~mm}$ radius in a polydimethylsiloxane matrix $\left(\eta_{m}=1 \mathrm{~Pa} \cdot \mathrm{s}, \quad \sigma=4 \mathrm{mN} / \mathrm{m}\right)$. The droplet has been extended in supercritical 2D hyperbolic flow, which causes a slightly different initial shape than the numerical "droplet" which is extended in an axisymmetrical flow. ${ }^{1}$ In shapes $2-5$, the flow is turned off, and the polybutadiene droplet quickly becomes axisymmetrical due to the interfacial tension. As is shown in Fig. 4, the droplet relaxation predicted by the numerical model correctly describes the actual droplet relaxation.

In general, the interfacial tension coefficient will not be constant if surfactants are present. The gradient in the interfacial tension coefficient is the result of a gradient in surfactant concentration along the interface. Therefore, one has to determine the surfactant distribution in order to determine the "volume" forces described above. To determine the surfactant distribution along the interface, a convection diffusion equation is solved for the concentration $\Gamma$. Li and Pozrikidis ${ }^{15}$ determined how this equation is defined on a moving mesh:

$$
\left(\frac{\partial \Gamma}{\partial t}\right)_{(\xi, \zeta)}+\Gamma \nabla_{s} \cdot \mathbf{v}-\mathcal{D}_{s} \nabla_{s}^{2} \Gamma=0
$$

where $D_{s}$ is the diffusivity. Since the equation is solved on a moving grid, the convective part of the equation is captured in the first term. The second term accounts for the change in concentration due to contraction/dilatation of the interface, and the third term of this equation is the diffusion term. Using the undeformed droplet radius $R$ as a characteristic length, $\dot{\epsilon}_{m} R$ as a characteristic velocity, and $\eta_{m} R / \sigma$ as a characteristic time, the equation can be nondimensionalized as

$$
\frac{1}{\mathrm{Ca}}\left(\frac{\partial \Gamma}{\partial t}\right)_{(\xi, \zeta)}+\Gamma \nabla_{s} \cdot \mathbf{v}-\frac{1}{\mathrm{Pe}} \nabla_{s}^{2} \Gamma=0,
$$

where the capillary number $\mathrm{Ca}=R \eta_{m} \dot{\epsilon}_{m} / \sigma$ equals the ratio between the deforming viscous forces and the restoring capillary forces. The Péclet number Pe equals $\mathrm{Pe}=\dot{\epsilon}_{m} R^{2} / \mathcal{D}_{s}$ and represents the ratio between convection and diffusion of the surfactants.

For surfactants without interactions between the molecules, the interfacial tension coefficient can be described by the Langmuir equation of state: ${ }^{7,8,22}$

$$
\sigma=\sigma_{0}+R_{g} T \Gamma_{\infty} \ln \left(1-\frac{\Gamma}{\Gamma_{\infty}}\right),
$$

where $\sigma_{0}$ equals the interfacial tension coefficient for a clean interface, $R_{g}$ is the gas constant, and $T$ the absolute temperature. The concentration $\Gamma_{\infty}$ is the maximum concentration possible (each surfactant molecule occupies a finite space on the interface). In terms of the dimensionless interfacial tension coefficient $\sigma^{*}=\sigma / \sigma_{r}$ and the dimensionless surfactant concentration $\Gamma^{*}=\Gamma / \Gamma_{r}$, Eq. (7) can be written as

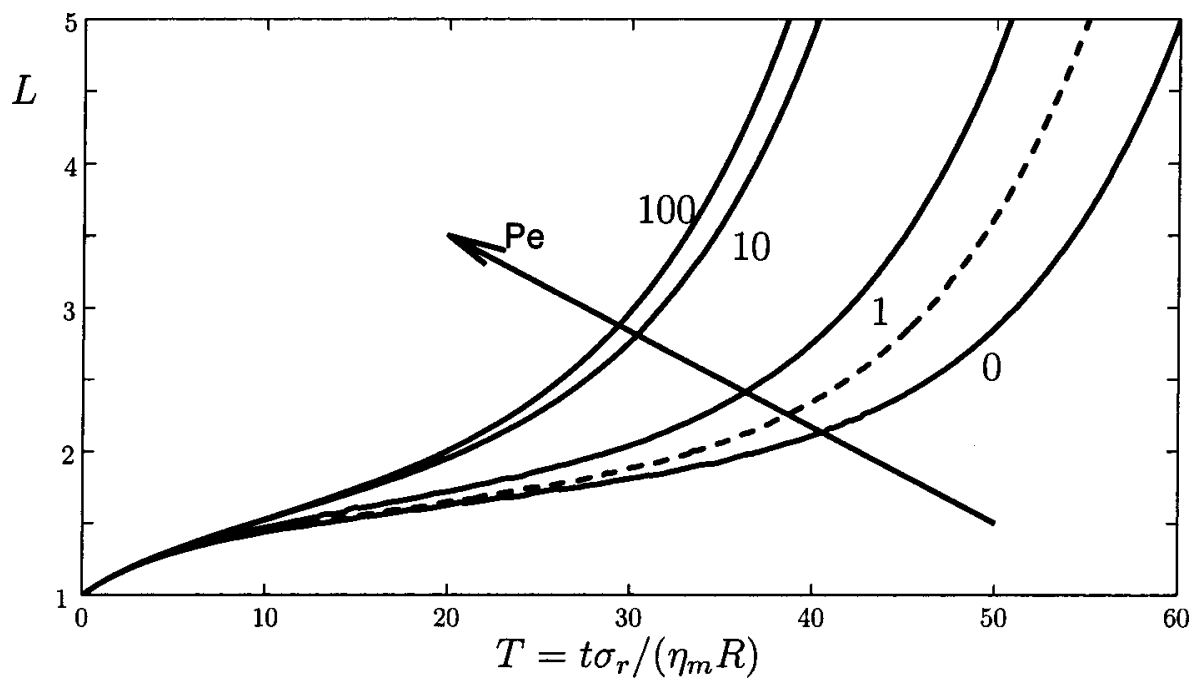

FIG. 6. The influence of the Péclet number Pe on the droplet deformation as a function of time, for $\beta=0.2, \Gamma_{r}$ $\ll \Gamma_{\infty}$, and $\mathrm{Ca}=0.1$. The dashed line represents the droplet deformation of a clean droplet, whereas the solid lines correspond to $\mathrm{Pe}=0,1,10$, and 100 . 
a) Langmuir relationship $\sigma^{*}\left(\Gamma^{*}\right)$

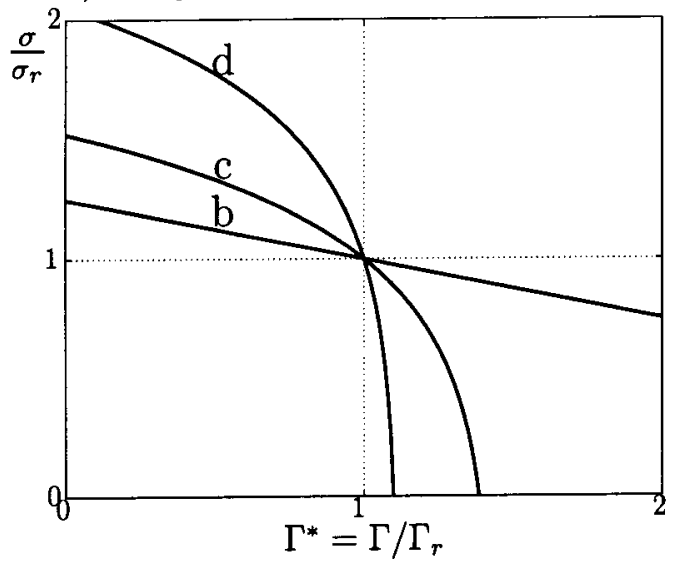

c) Results for $\Gamma_{r}=0.7 \Gamma_{\infty}, \beta=0.2$

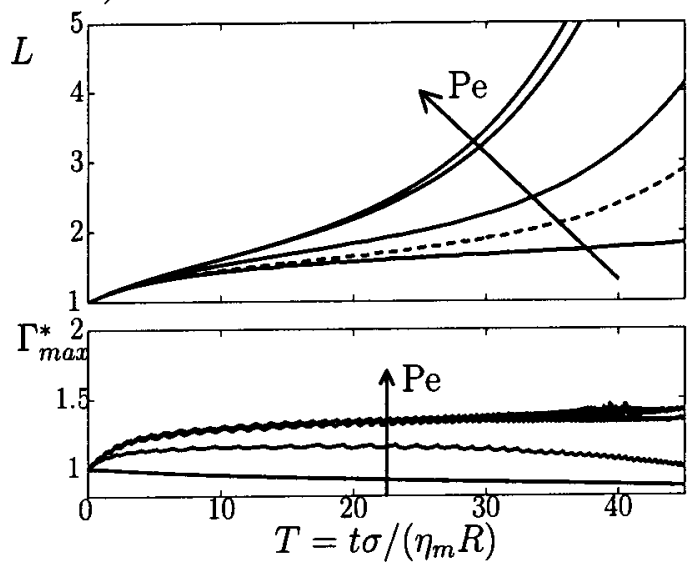

b) Results for $\Gamma_{r}=0.01 \Gamma_{\infty}, \beta=0.2$

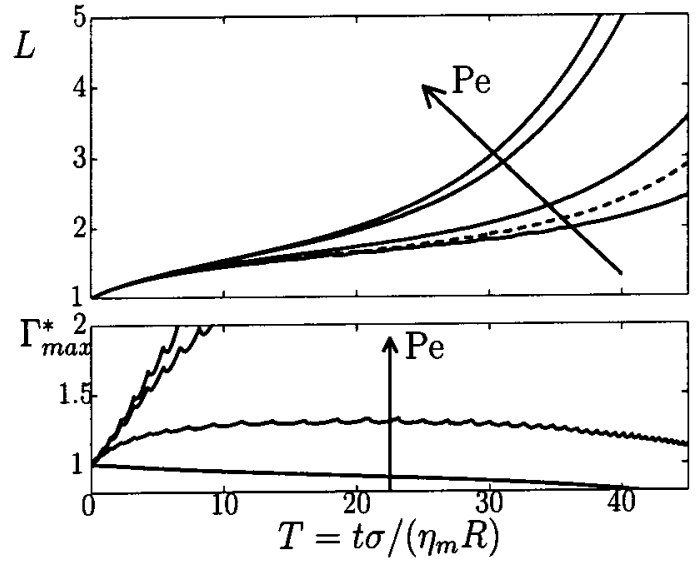

d) Results for $\Gamma_{r}=0.9 \Gamma_{\infty}, \beta=0.2$

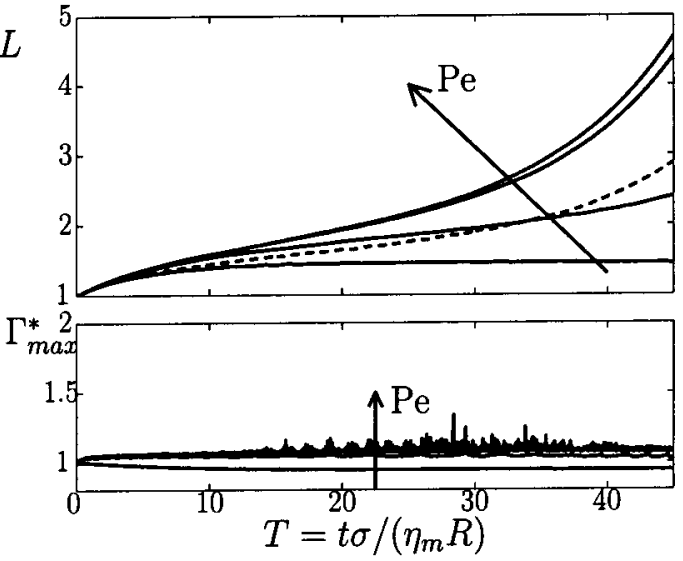

FIG. 7. Dimensionless droplet length $L$ and maximum concentration $\Gamma_{\max }$ as a function of dimensionless time $T$ at various Péclet numbers. The relationships between $\Gamma$ and $\sigma$ are given by the Langmuir equation of state Eq. (8): $\beta=0.2$ and (b) $\Gamma_{r}=0.01 \Gamma_{\infty}$, (c) $\Gamma_{r}=0.7 \Gamma_{\infty}$, and (d) $\Gamma_{r}=0.9 \Gamma_{\infty}$, see top left figure (a). The solid lines correspond to $\mathrm{Pe}=0,1,10$, and 100 , respectively, whereas the dashed line represents a clean droplet.

$$
\sigma^{*}=\frac{\sigma}{\sigma_{r}}=\frac{\frac{\Gamma_{r}}{\Gamma_{\infty}}+\beta \ln \left(1-\frac{\Gamma_{r}}{\Gamma_{\infty}} \Gamma^{*}\right)}{\frac{\Gamma_{r}}{\Gamma_{\infty}}+\beta \ln \left(1-\frac{\Gamma_{r}}{\Gamma_{\infty}}\right)} \quad \text { with } \beta=\frac{R_{g} T \Gamma_{r}}{\sigma_{0}},
$$

where $\sigma_{r}$ denotes the interfacial tension coefficient at the reference concentration $\Gamma_{r}$. This reference concentration equals the initial uniform concentration of surfactant on the undeformed droplet.

As described, the interfacial tension coefficient $\sigma$ is a function of the surfactant concentration. Therefore, $\sigma$ in Eq. (4) is replaced with $\sigma(r, z)$, such that the volume force on the total element is given by

$$
\begin{aligned}
\mathbf{f}_{v}= & \frac{2 \sigma_{5} r_{5}\left[\sin (\gamma) \mathbf{e}_{r}+\cos (\gamma) \mathbf{e}_{z}\right]}{d z\left(2 d r R_{b}+d r^{2}\right)} \\
& -\frac{2 \sigma_{2} r_{2}\left[\sin (\vartheta) \mathbf{e}_{r}+\cos (\vartheta) \mathbf{e}_{z}\right]}{d z\left(2 d r R_{b}+d r^{2}\right)} \\
& -\frac{2\left[\sigma_{23} l_{23}+\sigma_{34} l_{34}+\sigma_{45} l_{45}\right] \mathbf{e}_{r}}{d z\left(2 d r R_{b}+d r^{2}\right)},
\end{aligned}
$$

where $R_{b}$ is the radius at the bottom of the mesh segment and $d r$ the segments height, whereas the angles $\vartheta$ and $\gamma$ are defined in Fig. 5. The open circles in Fig. 5 denote temporary points, which are used solely for the calculation of volume force and are deleted afterwards. Their interfacial tension coefficient is calculated from their neighboring marker points: $\sigma_{2}=\sigma_{1} l_{12} / l_{13}+\sigma_{3} l_{23} / l_{13}$. Due to the varying interfacial tension coefficient, the volume forces are no longer directed perpendicular to the surface. Therefore the volume forces can no longer be countered by a pressure jump over the interface alone. Instead, the tangential component of the force also gives rise to an additional velocity along the interface.

The velocity field is calculated using a Petrov-Galerkin finite element method. ${ }^{23}$ Both the velocity field $\mathbf{v}$ and the volume forces $\mathbf{f}_{v}$ (due to the interfacial tension) are approximated with biquadratic shape functions. As bilinear interpolation functions are used for the pressure $p$, the Ladyzhenskaya-Babuška-Brezzi conditions ${ }^{24,25}$ are satisfied and a unique solution of the Stokes equations is guaranteed. Although the method is in principle suitable for any computational domain, the simulations in this study have been limited to the deformation and breakup in axisymmetrical sys- 
a) Langmuir relationship $\sigma^{*}\left(\Gamma^{*}\right)$

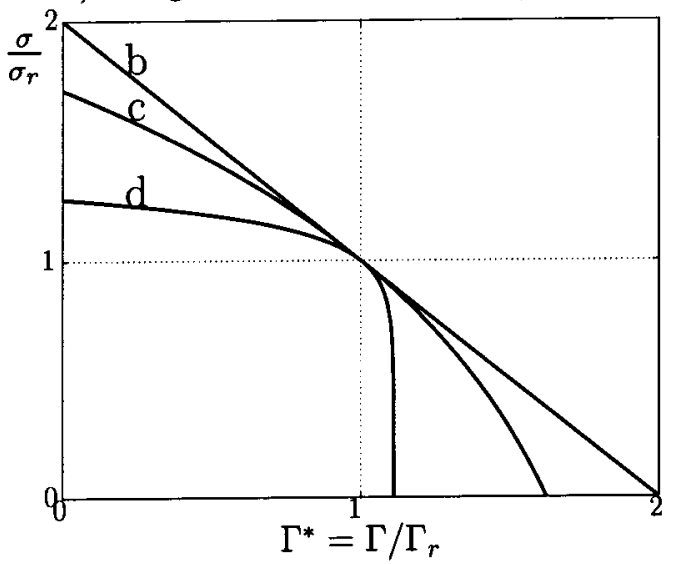

c) Results for $\Gamma_{r}=0.5 \Gamma_{\infty}, \beta=0.3$

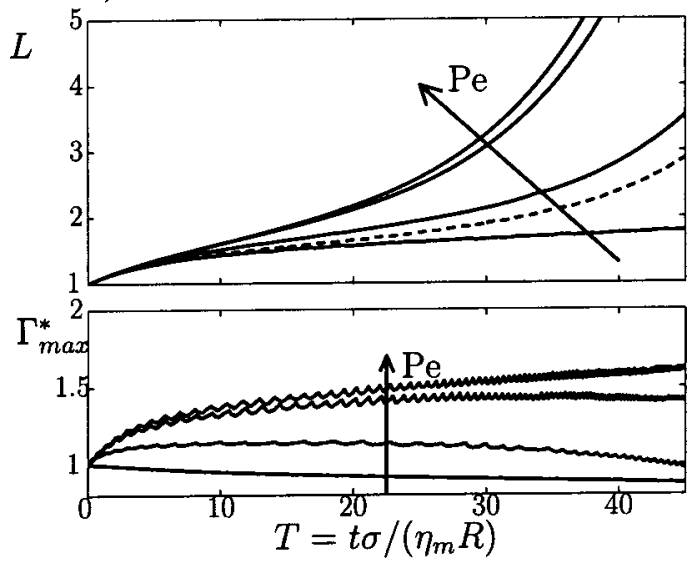

b) Results for $\Gamma_{r}=0.01 \Gamma_{\infty}, \beta=0.5$

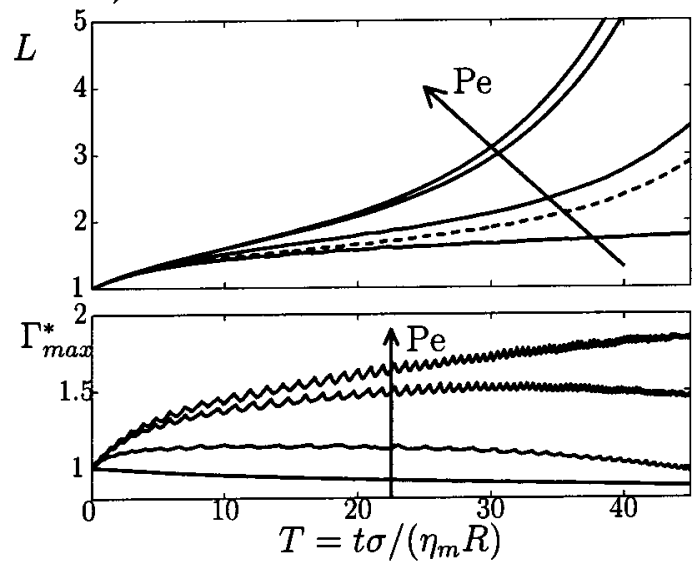

d) Results for $\Gamma_{r}=0.9 \Gamma_{\infty}, \beta=0.08$

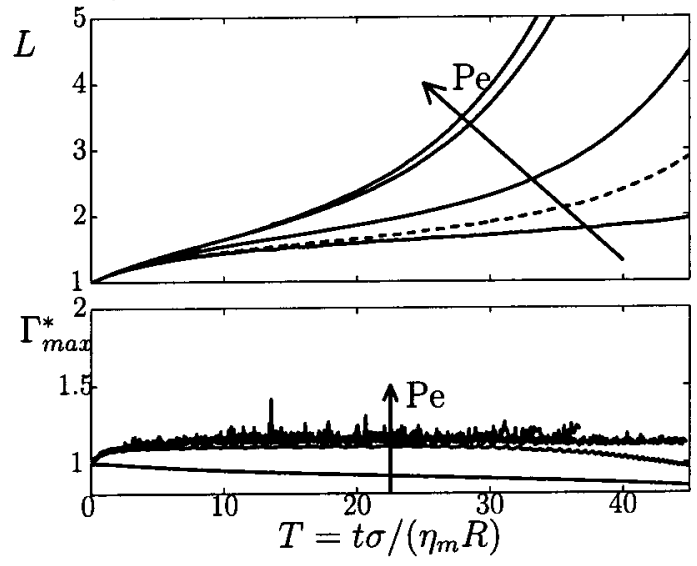

FIG. 8. Dimensionless droplet length $L$ and maximum concentration $\Gamma_{\max }$ as a function of dimensionless time $T$ at various Péclet numbers. The relationships between $\Gamma$ and $\sigma$ are given by the Langmuir equation of state, Eq. (8): (b) $\Gamma_{r}=0.01 \Gamma_{\infty}, \beta=0.5$, (c) $\Gamma_{r}=0.5 \Gamma_{\infty}, \beta=0.3$, and (d) $\Gamma_{r}=0.9 \Gamma_{\infty}, \beta=0.08$. For these parameters, $\partial \sigma / \partial \Gamma$ at the reference state is equal for all relationships, see top left figure (a). The solid lines correspond to $\mathrm{Pe}=0,1,10$, and 100 , respectively, whereas the dashed line represents a clean droplet.

tems to decrease the requirements on CPU and memory usage. Due to the axisymmetry and the fact that only half the droplet $(z>0)$ is simulated, the $r$ - and $z$-axes are symmetry axes. On the other two edges of the domain, Dirichlet boundary conditions are prescribed: $v_{r}=-\frac{1}{2} \dot{\varepsilon}_{m} r$ and $v_{z}=\dot{\varepsilon}_{m} z$. The pressure $p$ is set to zero at the corner opposite to the origin. Initially, all unknowns are set to zero, which corresponds to a quiescent fluid. Although the method described above is general, the results in this study have been obtained by integrating user-written Fortran subroutines ${ }^{1}$ into the finite element package SEPRAN (Ref. 26) using a SPARSKIT (Ref. 27) solver.

\section{RESULTS}

The influence of surfactant on the time dependent droplet deformation in supercritical flows is investigated for various values of the Péclet number. To reduce the number of parameters, the investigation is limited to an equal viscosity system and to a single supercritical capillary number, $\mathrm{Ca}$ $=0.1$.

\section{A. Influence of Péclet number}

The influence of the Péclet number on the droplet deformation in a supercritical flow for a low concentration $\left(\Gamma_{r}\right.$ $\ll \Gamma_{\infty}$ ) of a surfactant with $R_{g} T \Gamma_{r} / \sigma_{0}=0.2$ is shown in Fig. 6 . The results are similar to those described in the previous section for subcritical flow.

For $\mathrm{Pe} \ll 1$ (negligible convection), the droplet deformation curve of a surfactant covered droplet is below the curve for a clean droplet with a (constant) interfacial tension coefficient $\sigma_{r}$. This is due to the fact that for $\mathrm{Pe} \ll 1$, the surfactant concentration is uniform over the interface. The deformation is therefore dilution dominated, i.e., the interfacial tension coefficient increases in time due to an increase in interfacial area of the droplet. Therefore, the effective capillary number experienced by the droplet decreases with time, even though the flow field is constant. In this diffusion dominated case, the deformation of a surfactant covered droplet in a steady elongational flow can in fact be fully described by considering a clean droplet with $\sigma=\sigma_{r}$ in a time dependent 
a) Langmuir relationship $\sigma^{*}\left(\Gamma^{*}\right)$

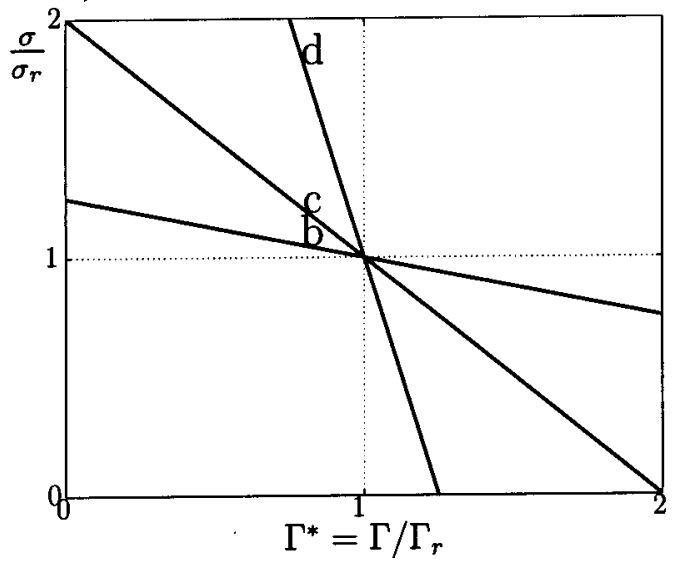

c) Results for $\beta=0.5$

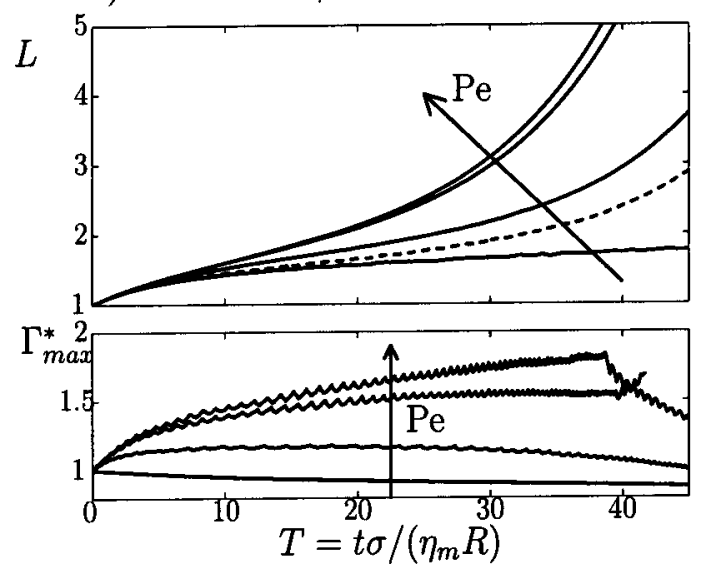

b) Results for $\beta=0.2$

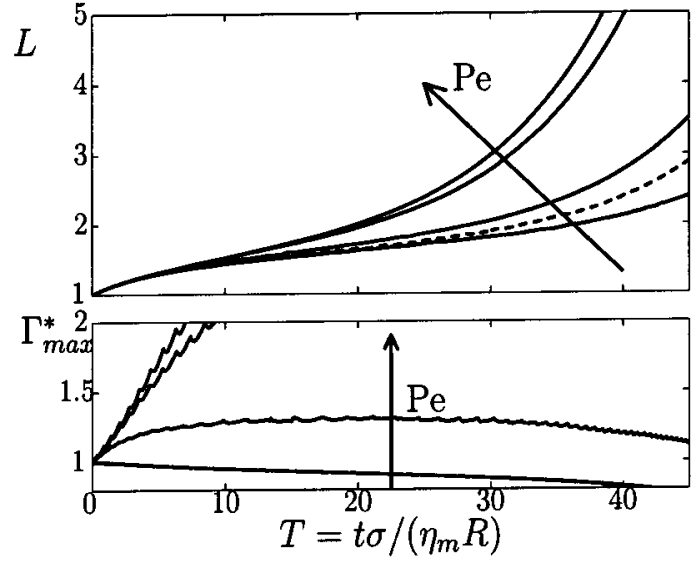

d) Results for $\beta=0.8$

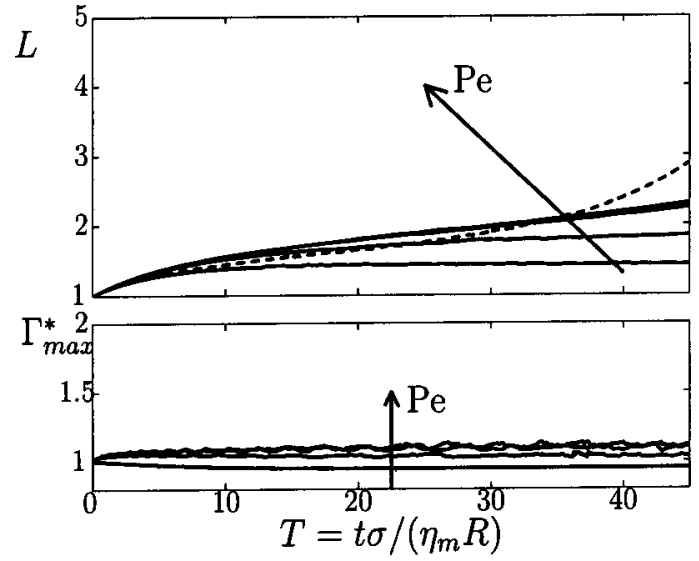

FIG. 9. Dimensionless droplet length $L$ and maximum concentration $\Gamma_{\max }$ as a function of dimensionless time $T$ at various Péclet numbers. The relationships between $\Gamma$ and $\sigma$ are given by the equation of state Eq. (8): (b) $\beta=0.2$, (c) $\beta=0.5$, and (d) $\beta=0.8$, see top left figure (a). The solid lines correspond to Pe=0, 1,10 , and 100 , respectively, whereas the dashed line represents a clean droplet.

flow given by $\mathrm{Ca}(t)=\mathrm{Ca}_{0} A_{0} / A$ with $A$ the instantaneous interfacial area of the droplet.

If convection is not negligible, the situation becomes more complicated. Figure 6 shows that the droplet deformation at a given dimensionless time increases with increasing Péclet number. This is due to the fact that with increasing Péclet number the surfactant distribution over the droplet becomes increasingly inhomogeneous. Due to the convection, the surfactant accumulates at the tips of the droplet, whereas the surfactant concentration in the central region decreases. The increase in surfactant concentration at the tips causes a decrease in the local interfacial tension coefficient. Therefore, the deformation has to increase at the tips in order to maintain the balance between the interfacial and the viscous forces.

From the above, it is clear that for low concentrations of this surfactant, the droplet deformation at a given time is larger than the deformation of a clean drop if the convection is dominant, whereas it is smaller if diffusion dominates. However, this is not necessarily the case for higher concentrations and/or different types of insoluble surfactant.

\section{B. Influence of concentration for constant $\beta$}

Figure 7 shows the deformation of droplets which are covered with different concentrations of surfactant. The relations between the interfacial tension coefficient and the surfactant concentration are given by the Langmuir equation of state Eq. (8), see Fig. 7(a). For all three relations the surfactant is described by $R_{g} T \Gamma_{r} / \sigma_{0}$ is 0.2 , but the surfactant coverage $\Gamma_{r} / \Gamma_{\infty}$ changes. $^{28}$

Figure 7(a) shows the relation between the dimensionless surfactant concentration $\Gamma^{*}$ and the dimensionless interfacial tension coefficient $\sigma^{*}$. As the upper limit of the concentration $\Gamma_{\infty}$ is approached, the interfacial tension coefficient drops fast and relative small gradients in the concentration cause huge gradients in the interfacial tension coefficient. Therefore, the Marangoni stresses which oppose the surfactant convection become very large, ensuring that $\Gamma_{\infty}$ will be not reached. ${ }^{29}$

If diffusion is dominant, there are no concentration gradients and the dimensionless concentration $\Gamma^{*}=\Gamma / \Gamma_{r}$ equals $\Gamma_{\max }^{*}$ given in Figs. 7(b)-7(d). At any given dimensionless 

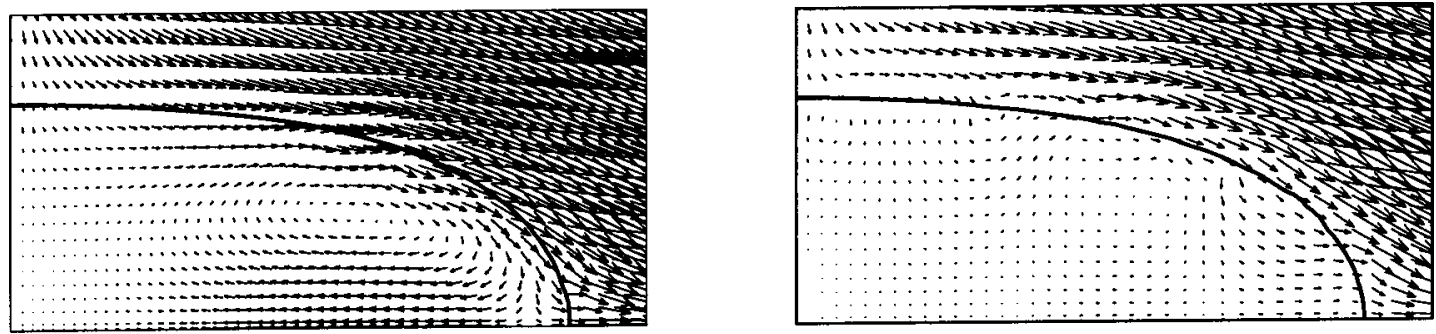

FIG. 10. Velocity fields for surfactant covered droplets with $\beta=0.2, \mathrm{Pe}=0$ (left) and $\beta=0.8, \mathrm{Pe}=1$ (right), respectively. The surfactant distribution is in both cases homogeneous. However, for $\beta=0.2$, the homogeneous surfactant distribution is due to diffusion, whereas for $\beta=0.8$, it is due to Marangoni stresses. Therefore, internal circulation is present for $\beta=0.2$, but not for $\beta=0.8$. The droplet deformation $L$ equals 1.8 .

time, the dimensionless concentration $\Gamma^{*}$ increases with decreasing $\Gamma_{r} / \Gamma_{\infty}$. However, since $\sigma^{*}\left(\Gamma^{*}\right)$ varies less for lower surfactant coverage, the highest droplet deformation is found for $\Gamma_{r} \ll \Gamma_{\infty}$.

Again, the situation is more complex in case of convection. Starting with a very low concentration of surfactant $\left(\Gamma_{r}=0.01 \Gamma_{\infty}\right)$, the deformation will first increase with increasing surfactant coverage [see Figs. 7(b) and 7(c)], due to the much higher dependence of $\sigma^{*}$ on $\Gamma^{*}$. However, if the surfactant coverage becomes very high $\left(\Gamma_{r}=0.9 \Gamma_{\infty}\right)$ the deformation of the droplet is smaller than for a droplet with $\Gamma_{r}=0.01 \Gamma_{\infty}$ for all Péclet numbers [see Fig. 7(d)]. This decrease in droplet deformation is caused by strong Marangoni stresses which keep the concentration almost constant over the interface. Therefore, dilution becomes a dominant effect and the droplet deformation is largely decreased.

We can therefore conclude that the droplet deformation in supercritical flow does not only depend on the Péclet number, but also on the concentration of the surfactant. This is in correspondence with the results obtained in (sub-)critical flow by $\mathrm{Hu}$ and Lips, ${ }^{8}$ Janssen et al. ${ }^{10,13}$ Eggleton et al. ${ }^{12}$ and Velankar et al. ${ }^{14}$ It remains to be seen, however, whether this should be attributed to the change in $\partial \sigma^{*} / \partial \Gamma^{*}$ around the reference concentration, or due to the proximity of $\Gamma_{\infty}^{*}$. Therefore, these cases will be considered separately.

\section{Influence of concentration for constant $\partial \sigma / \partial \Gamma$}

We consider droplets which are covered with various concentrations of surfactants described by the Langmuir equation, Eq. (8). All droplets have the same reference interfacial tension coefficient. Moreover, the dependence $\partial \sigma^{*} / \partial \Gamma^{*}$ for concentrations close to the reference concentration $\Gamma_{r}$ is also equal, see Fig. 8(a). These properties can only be obtained if different surfactants (with different $\Gamma_{\infty}$ ) are used, since $\beta$ has to decrease with increasing $\Gamma_{r} / \Gamma_{\infty}$. Three combinations have been selected: $\left(\Gamma_{r}=0.01 \Gamma_{\infty}, \beta=0.5\right)$, $\left(\Gamma_{r}=0.5 \Gamma_{\infty}, \beta=0.3\right)$, and $\left(\Gamma_{r}=0.9 \Gamma_{\infty}, \beta=0.08\right)$. For all three combinations, the deformation of a droplet subjected to the supercritical flow is determined as a function of time for different Péclet numbers. Figure 8 shows that for a given droplet length $L=l / l_{0}$, the maximum concentration $\Gamma_{\max }^{*}$ decreases with increasing surfactant coverage $\Gamma_{r} / \Gamma_{\infty}$. This is due to the Marangoni stresses, which become high if the concentration $\Gamma^{*}$ approaches its upper limit $\Gamma_{\infty} / \Gamma_{r}$. Although the Marangoni stresses ensure that the upper limit is not reached, the local concentration becomes high enough to cause a severe reduction in interfacial tension coefficient. Therefore, the droplet deformation increases with increasing surfactant coverage $\Gamma_{r} / \Gamma_{\infty}$ in all convection dominated situations $\mathrm{Pe} \geqslant 1$, as is shown in Fig. 8. Apparently, the surfactant coverage $\Gamma_{r} / \Gamma_{\infty}$ is not the dominant factor for the effect described in the previous section.

\section{Influence of $\partial \sigma / \partial \Gamma$ for constant concentration}

If we consider low concentrations of surfactants, the Langmuir equation of state becomes linear. Figure 9 gives the deformation as a function of time for droplets with low concentrations of surfactant for various Péclet numbers. Three different values of $\beta$ are selected, which give the same dependence $\partial \sigma^{*} / \partial \Gamma^{*}$ around the reference state as the surfactants used in Fig. 7. Our results in supercritical flow resemble the results obtained by $\mathrm{Li}$ and Pozrikidis ${ }^{15}$ and Stone and $\mathrm{Leal}^{16}$ in subcritical flows. For $\mathrm{Pe}=0$, the droplet deformation decreases with increasing $\partial \sigma^{*} / \partial \Gamma^{*}$, i.e., with increasing $\beta$. For higher Péclet numbers $(\mathrm{Pe}>1)$, the deformation is higher for $\beta=0.5$ than for $\beta=0.2$, due to the decrease in interfacial tension coefficient near the tips of the droplet. However, for $\beta=0.8$, the Marangoni effects become so strong, that the droplet deformation becomes dilution dominated again, causing a smaller droplet deformation than in

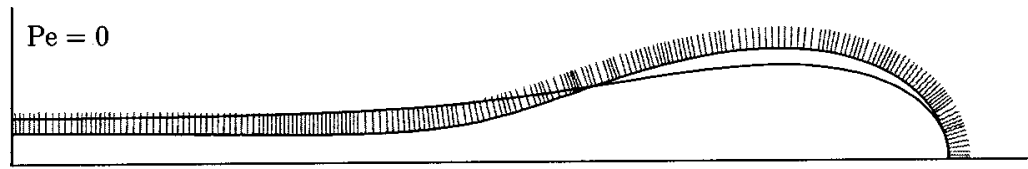

$\mathrm{Pe}=100$

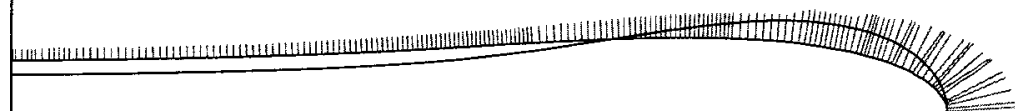

FIG. 11. Droplet shape and surfactant distribution for $\mathrm{Pe}=0$ (top) and $\mathrm{Pe}=100$ (bottom), with $\beta=0.5$ and $\Gamma_{r}$ $\ll \Gamma_{\infty}$. The droplets are obtained after 90.8 and 38.5 time units, respectively, at $\mathrm{Ca}=0.1$. The droplet shape of a clean droplet with $\sigma=\sigma_{r}$ is shown as a reference. The lines perpendicular to the interface indicate the surfactant concentration in the marker points. For $\mathrm{Pe}=0$, the concentration is constant at $58 \%$ of the initial concentration $\Gamma_{r}$. For $\mathrm{Pe}=100$ the concentration varies between $0.32 \Gamma_{r}$ and $1.82 \Gamma_{r}$. 

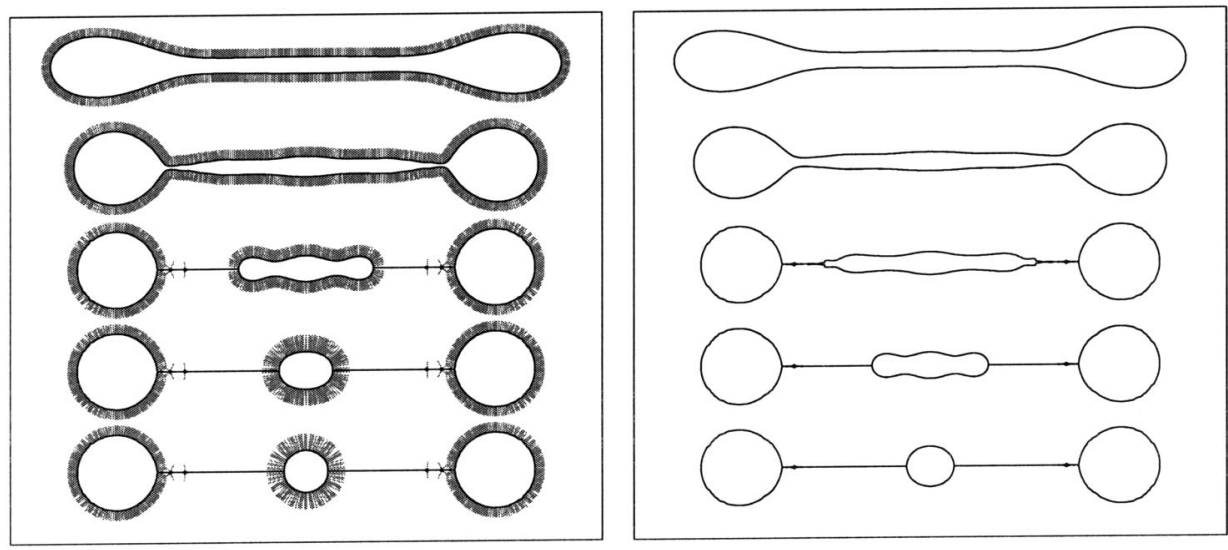

FIG. 12. Breakup of an elongated droplet with $L_{0}=5$. The left droplet is covered with surfactant $(\beta=0.5$, $\mathrm{Pe}=0)$ and is obtained at $\mathrm{Ca}=0.1$. The right droplet starts with the same initial shape, but has a constant interfacial tension coefficient $\sigma=\sigma_{r}$. Shapes are given for $T=0,5,10,15$, and 20 . The lines perpendicular to the interface indicate the surfactant concentration in the marker points (Ref. 31). The interfacial tension coefficient of the surfactant covered daughter droplets is $0.89 \sigma_{r}$ for the central droplet and $1.42 \sigma_{r}$ for the outer droplets. the corresponding case of a clean droplet. Therefore, the influence of (bulk) surfactant concentration on droplet deformation shown in Fig. 7 seems to be caused by the increased dependence of the interfacial tension coefficient $\sigma^{*}$ on the concentration $\Gamma^{*}$, and does not necessarily depend on the actual value of $\Gamma_{r} / \Gamma_{\infty}$.

Experimentally, the presence of Marangoni stresses is most easily observed by the absence of internal circulation. Figure 10 shows the velocity fields of two droplets with the same droplet length. The deformation of both droplets is dilution dominated, but the physical background of the homogeneous distribution is different. In the first droplet, $\beta=0.2$ and $\mathrm{Pe}=0$, the distribution is homogeneous because diffusion is dominant, whereas in the second droplet, $\beta=0.8$ and $\mathrm{Pe}=1$, the homogeneous distribution is due to Marangoni stresses. Whereas in the diffusion dominated case internal circulation is observed, this is not the case if high Marangoni stresses are present.

\section{E. Breakup of surfactant covered droplets in a quiescent matrix}

In the remainder of this paper, we focus on the breakup of elongated droplets after cessation of flow. Two issues are considered: the difference in breakup between a clean droplet and a surfactant covered droplet, and the influence of the Péclet number during extension. We use the Langmuir equation of state for low coverage $\Gamma_{r} \ll \Gamma_{\infty}$ and $\beta=0.5$ which gives a large difference in droplet deformation behavior between the convection dominated case and the diffusion dominated case.

The effect of the Péclet number on the droplet shape is shown in Fig. 11. For increasing Péclet number, the surfactant distribution over the droplet interface becomes increasingly inhomogeneous. Therefore, the interfacial tension coefficient is locally decreased near the tips of the droplet, which causes the increased curvature for higher Péclet numbers. Figures 12 and 13 show the breakup of the droplets shown in Fig. 11. They are compared with the breakup of droplets with the same elongated shape but with a clean interface. ${ }^{30}$ As expected, the surfactant has little influence on the breakup of a droplet if the surfactant distribution is homogeneous $(\mathrm{Pe}=0)$. In this case, the only difference between the breakup of the surfactant covered droplet and the clean droplet is the time scale of the process. Whereas the clean droplet has a constant interfacial tension coefficient $\sigma=\sigma_{r}$, the interfacial tension coefficient of the surfactant covered droplet is initially $1.42 \sigma_{r}$ and decreases with decreasing interfacial area. Therefore, the relaxation of the surfactant covered droplet is initially much faster. In both situations, however, three daughter droplets with equal size distributions result. Only if these daughter droplets would be subjected to flow, a difference in behavior would be obvious, since the clean droplets would still have interfacial tension coefficient $\sigma_{r}$, whereas the interfacial tension coefficient of the surfac-
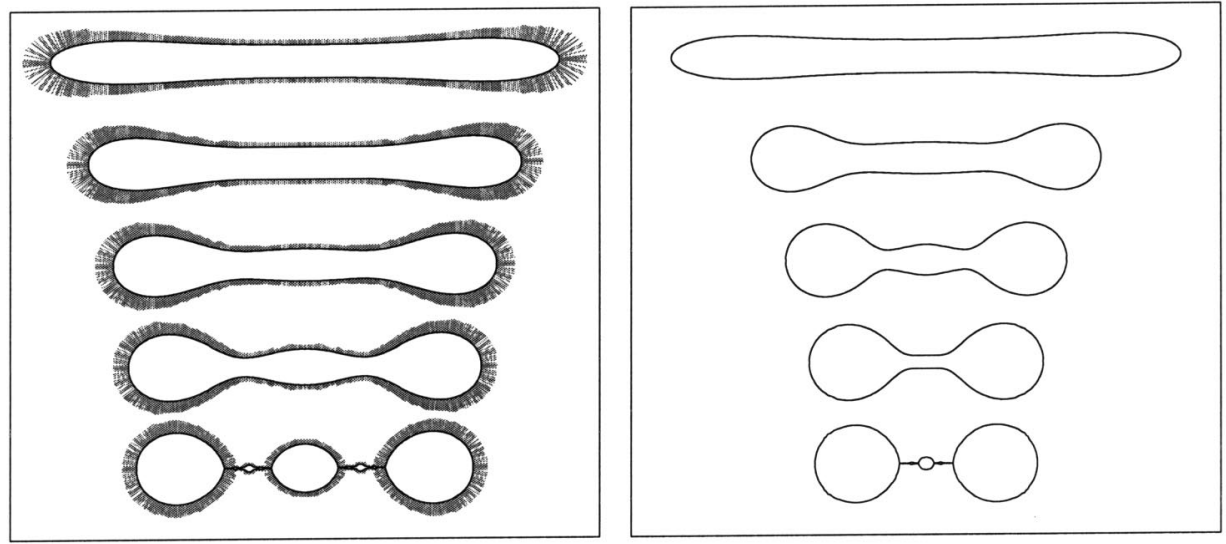

FIG. 13. Breakup of an elongated droplet with $L_{0}=5$. The left droplet is covered with surfactant $(\beta=0.5, \mathrm{Pe}$ $=100)$ and is obtained at $\mathrm{Ca}=0.1$. The right droplet starts with the same initial shape, but has a constant interfacial tension coefficient $\sigma=\sigma_{r}$. Shapes are given for $T=0,5,10,15$, and 20 . The lines perpendicular to the interface indicate the surfactant concentration in the marker points (Ref. 31). The interfacial tension coefficient of the surfactant covered daughter droplets is $1.62 \sigma_{r}$ for the central droplet and $1.20 \sigma_{r}$ for the outer droplets. 


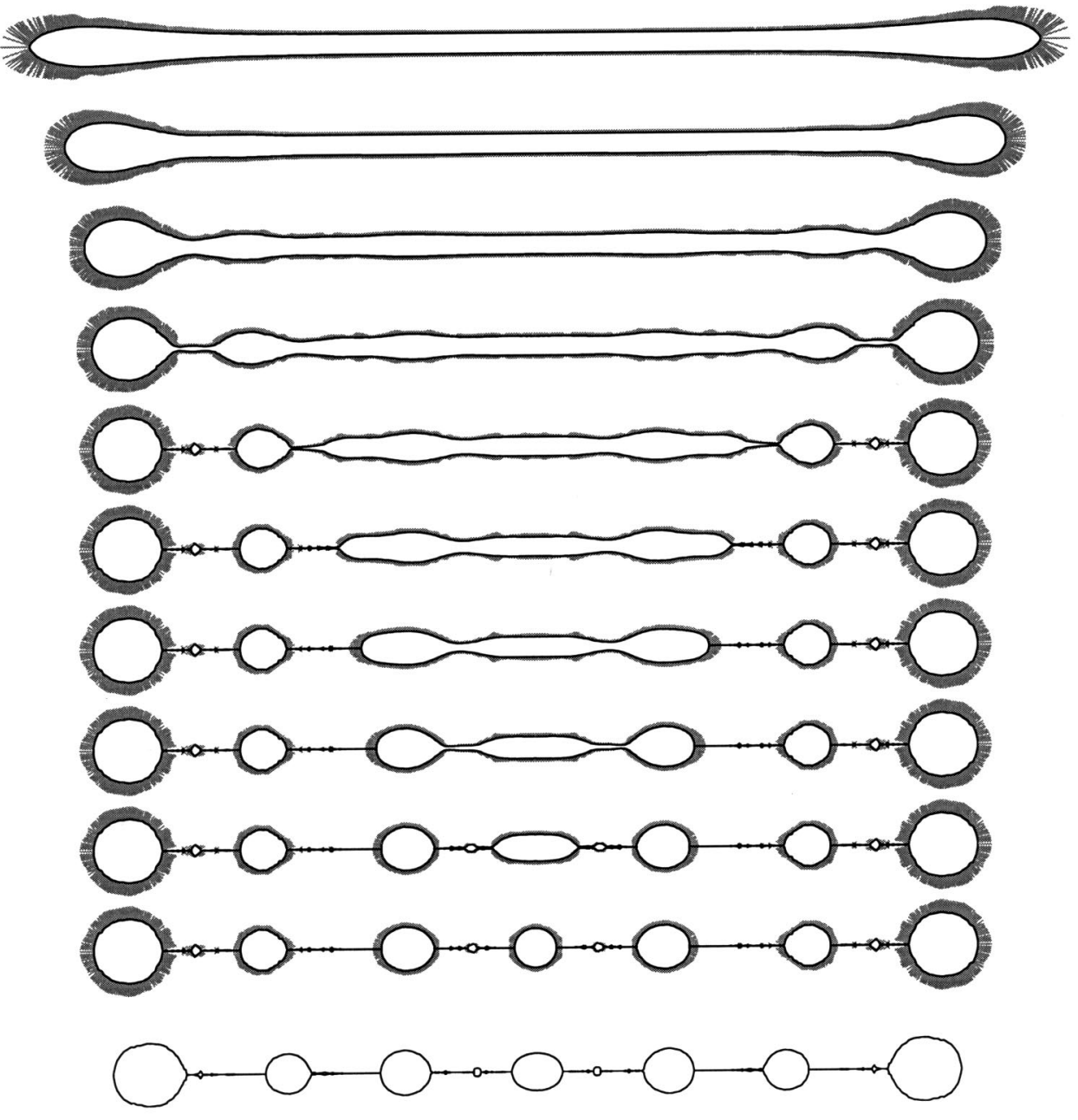

FIG. 14. Breakup of a highly elongated droplet with $L_{0}=10$ covered with surfactant $(\beta=0.5)$. The initial droplet shape is obtained at $\mathrm{Ca}=0.1$ and $\mathrm{Pe}=100$. Given droplet shapes are not equally spaced in time. The lines perpendicular to the interface indicate the concentration in the marker points (Ref. 31). The interfacial tension coefficient of the daughter droplets varies from $1.19 \sigma_{r}$ (outer droplets) to $1.70 \sigma_{r}$ (central droplet). At the bottom, the final droplet distribution after breakup of a clean droplet with the same initial shape as the surfactant covered droplet is given.

tant covered droplets would be either increased to $1.42 \sigma_{r}$ (outer two droplets) or decreased to $0.89 \sigma_{r}$ (central droplet). This effect has also been observed experimentally by $\mathrm{Hu}$ et al. $^{17}$

If the "initial" distribution of the surfactant is nonhomogeneous, the surfactant has more influence on the breakup process, as is shown in Fig. 13. Due to the relative high interfacial tension coefficient near the necks, the droplet has less time to relax in the case of the surfactant covered drop. Therefore, this droplet breaks into three daughter droplets, whereas the clean droplet with the same "initial" shape breaks into two daughter droplets (+ satellite droplets).

This is in contradiction with the statement by Milliken et $a l .{ }^{4}$ that the initial surfactant distribution hardly affects the breakup behavior of moderately extended droplets. On the other hand, Milliken et al. ${ }^{4}$ observed a distinct difference in breakup behavior between a highly extended surfactant covered droplet and a clean droplet with the same "initial" shape. This difference is in turn not observed during our simulations of such a highly extended droplet obtained at $\mathrm{Ca}=0.1$ with $\mathrm{Pe}=100$. As is shown in Fig. 14, the exact same distribution of the daughter droplets results. Also during the breakup process, the same droplet shapes were observed, and the only differences which could be found were the breakup time (about twice as large in case of the clean droplet with $\sigma=\sigma_{r}$ ) and the interfacial tension coefficient of the daughter droplets.

\section{DISCUSSION AND CONCLUSIONS}

The droplet deformation and breakup of surfactant covered droplets have been studied. To limit the number of variables, only droplets in an equal viscosity system at $\mathrm{Ca}=0.1$ have been considered, while varying the Péclet number, the surfactant coverage $\Gamma_{r} / \Gamma_{\infty}$, and the interaction parameter $\beta$. In general, the influence of the Péclet number on the droplet deformation in supercritical flows $(\mathrm{Ca}=0.1)$ was found to be similar to its influence on the droplet deformation in subcritical flows. Following Eggleton et al., ${ }^{12}$ we investigated the influence of the surfactant coverage on the deformation of a droplet covered with a surfactant described by the Langmuir equation of state with $\beta=0.2$. Similar to the results obtained by Eggleton et al. ${ }^{12}$ in subcritical flow, the droplet deformation was found to increase with increasing surfactant coverage $\Gamma_{r} / \Gamma_{\infty}$ for low and moderate $\Gamma_{r} / \Gamma_{\infty}$. Please note that this is not due to some overall decrease of the interfacial tension coefficient, because the interfacial tension coefficient in the reference state is equal in all cases. The increased droplet deformation is due to the increased dependence of the interfacial tension coefficient on the surfactant concentra- 
tion at higher surfactant coverage. If the distribution of the surfactant over the interface is the same, the interfacial tension coefficient at the tips of the droplet with a surfactant coverage $\Gamma_{r}=0.7 \Gamma_{\infty}$ will therefore be lower than for a droplet with surfactant coverage $\Gamma_{r}=0.01 \Gamma_{\infty}$.

As the surfactant coverage becomes very high $\left(\Gamma_{r}\right.$ $=0.9 \Gamma_{\infty}$ ), the interfacial tension coefficient becomes highly dependent on the surfactant concentration. Therefore, relatively small gradients in the concentration cause large Marangoni stresses. These Marangoni stresses prevent the accumulation of surfactant near the tips, causing the local interfacial tension coefficient at high surfactant coverage $\left(\Gamma_{r}=0.9 \Gamma_{\infty}\right)$ to remain higher than at the tips of droplets with $\Gamma_{r} \leqslant 0.7 \Gamma_{\infty}$.

It has been shown that these effects are mainly caused by the increased dependence of the interfacial tension coefficient on the surfactant concentration due to the constant value of $\beta$, instead of the actual value of $\Gamma / \Gamma_{\infty}$, by considering two other cases where one of these properties is kept constant while the other is varied.

Droplet breakup in a quiescent matrix has been studied for trace concentrations $\left(\Gamma_{r} \ll \Gamma_{\infty}\right)$ of an insoluble surfactant with $\beta=0.5$. Two droplets have been extended to $L=5$ at capillary number $\mathrm{Ca}=0.1$ with Péclet number 0 and 100 , respectively. At this capillary number, the droplet with $\mathrm{Pe}=0$ develops a distinct neck, whereas neck formation for the droplet with $\mathrm{Pe}=100$ is less obvious. Whereas both the surfactant covered droplets $(\mathrm{Pe}=0$ and $\mathrm{Pe}=100)$ break up into three droplets upon cessation of the flow, clean droplets with the same extended shapes will break into three and two droplets, respectively. The daughter droplet of the surfactant covered droplets have a different interfacial tension coefficient than the parent droplets. If the surfactant concentration prior to breakup is homogeneous $(\mathrm{Pe}=0)$, the interfacial tension coefficient is lower for the outer two droplets and higher for the central droplet. If the surfactant was accumulated at the tips $(\mathrm{Pe}=100)$, the opposite is true.

For a highly elongated droplet $(L=10)$ extended at $\mathrm{Ca}$ $=0.1$ with $P e=100$, no difference was found in the breakup process upon cessation of flow. The size distribution of the daughter droplets resulting from the surfactant covered droplet and the clean droplet are equal, and the only difference is found in their interfacial tension coefficient.

In conclusion, we can state that surfactant has indeed an influence on the droplet deformation. Whether a surfactant covered droplet deforms more or less than a clean droplet depends on the surfactant used. Two processes compete: surfactant accumulation near the tips due to convection and overall surfactant dilution due to the increase of interfacial area. The first effect tends to increase the droplet deformation and becomes more important if $\mathcal{D}_{s}$ decreases. The second effect becomes dominant if the diffusion coefficient $\mathcal{D}_{s}$ is high $(\mathrm{Pe}=0)$, or if the influence of the surfactant concentration on the interfacial tension coefficient is very high (Marangoni stresses).

The most important effect of surfactant on the breakup of droplets is the fact that the daughter droplets resulting after breakup do not have the same interfacial tension coefficient. Some daughter droplets will have a larger interfacial tension coefficient than the parent droplet, whereas the interfacial tension coefficient of other droplets will be lower. Since most mixing processes involve a time dependent flow, the resulting droplets will usually be subjected to elongational or shear flow again. Depending on their interfacial tension coefficient, this flow will or will not be strong enough to cause breakup. Therefore, insoluble surfactants may lead to broad size distributions in practical mixing processes.

This effect can be reduced by using soluble surfactants, which will reduce the difference in surfactant concentration between the droplets. Whether the surfactant should be soluble in the droplet phase or in the matrix phase depends on various factors. According to Milliken and Leal, ${ }^{9}$ the effect of surfactants which are soluble in the matrix phase on the droplet deformation is similar to the effect of insoluble surfactants. The effect of solubility in the droplet phase is not yet studied. However, one would expect that this would change the deformation and especially the breakup process, since the amount of surfactant available to the interface becomes dependent on the droplet shape: the amount of surfactant available in the necking region will be much smaller than in the bulbous ends. Moreover, surfactant solubility has a large effect on coalescence, and may prevent phase inversion.

\section{ACKNOWLEDGMENTS}

This research was financially supported by the European Community/FAIR Agriculture and Fisheries, Grant No. CT 97 3022, as well as the Dutch Polymer Institute.

${ }^{1}$ Y. W. Stegeman, "Time dependent behavior of droplets in elongational flows," Ph.D. thesis, Eindhoven University of Technology, 2002.

${ }^{2}$ W. J. Milliken and L. G. Leal, "Deformation and breakup of viscoelastic drops in planar extension flows," J. Non-Newtonian Fluid Mech. 40, 355 (1991).

${ }^{3}$ D. C. Tretheway and L. G. Leal, "Surfactant and viscoelastic effects on drop deformation in 2D extensional flow," AIChE J. 45, 929 (1999).

${ }^{4}$ W. J. Milliken, H. A. Stone, and L. G. Leal, "The effect of surfactant on the transient motion of Newtonian drops," Phys. Fluids A 5, 69 (1993).

${ }^{5}$ W. Bartok and S. G. Mason, "Particle motions in sheared suspensions VIII. Singlets and doublets of fluid spheres," J. Colloid Sci. 14, 13 (1959).

${ }^{6}$ R. A. de Bruijn, "Tipstreaming of drops in simple shear flows," Chem. Eng. Sci. 48, 277 (1993).

${ }^{7}$ Y. Pawar and K. J. Stebe, "Marangoni effects on drop deformation in extensional flow: The role of surfactant physical chemistry. I. Insoluble surfactants," Phys. Fluids 8, 1738 (1996).

${ }^{8}$ Y. T. Hu and A. Lips, "Estimating surfactant surface coverage and decomposing its effect on drop deformation," Phys. Rev. Lett. 91, 044501 (2003).

${ }^{9}$ W. J. Milliken and L. G. Leal, "The influence of surfactant on the deformation and breakup of a viscous drop: The effect of surfactant solubility," J. Colloid Interface Sci. 166, 275 (1994).

${ }^{10}$ J. J. M. Janssen, A. Boon, and W. G. M. Agterof, "Influence of dynamic interfacial properties on droplet breakup in simple shear flow," AIChE J. 40, 1929 (1994)

${ }^{11}$ J. J. M. Janssen, A. Boon, and W. G. M. Agterof, "Influence of dynamic interfacial properties on droplet breakup in plane hyperbolic flow," AIChE J. 43, 1436 (1997).

${ }^{12}$ C. D. Eggleton, Y. P. Pawar, and K. J. Stebe, "Insoluble surfactants on a drop in an extensional flow: A generalization of the stagnated surface limit to deforming interfaces," J. Fluid Mech. 385, 79 (1999). 
${ }^{13}$ J. J. M. Janssen, A. Boon, and W. G. M. Agterof, "Droplet break-up in simple shear flow in the presence of emulsifiers," Colloids Surf. A 91, 141 (1994).

${ }^{14}$ S. Velankar, P. van Puyvelde, J. Mewis, and P. Moldenaers, "Effect of compatabilization on the breakup of polymeric drops in shear flow," J. Rheol. 45, 1007 (2001).

${ }^{15} \mathrm{X}$. Li and C. Pozrikidis, "The effect of surfactants on drop deformation and on the rheology of dilute emulsions in Stokes flow," J. Fluid Mech. 341, 165 (1997).

${ }^{16} \mathrm{H}$. A. Stone and L. G. Leal, "The effects of surfactants on drop deformation and breakup," J. Fluid Mech. 220, 161 (1990).

${ }^{17}$ Y. T. Hu, D. J. Pine, and L. G. Leal, "Drop deformation, breakup, and coalescence with compatibilizer," Phys. Fluids 12, 484 (2000).

${ }^{18}$ I. Fortelny, "Breakup and coalescence of dispersed droplets in compatibilized polymer blends," J. Macromol. Sci., Phys. B39, 67 (2000).

${ }^{19}$ W. Press, S. A. Teukolsky, and W. T. Vetterling, Numerical Recipes in Fortran (Cambridge University Press, Cambridge, 1992).

${ }^{20}$ O. S. Galaktionov, P. D. Anderson, G. W. M. Peters, and F. N. van de Vosse, "An adaptive front tracking technique for three-dimensional transient flows," Int. J. Numer. Methods Fluids 32, 201 (2000).

${ }^{21} \mathrm{P}$. D. Anderson, "Computational analysis of distributive mixing," Ph.D. thesis, Eindhoven University of Technology, 1999.

${ }^{22}$ C. D. Eggleton, T.-M. Tsai, and K. J. Stebe, "Tip streaming from a drop in the presence of surfactants," Phys. Rev. Lett. 87, 048302 (2001).

${ }^{23}$ A. C. B. Bogaerds, "Stability analysis of viscoelastic flows," Ph.D. thesis, Eindhoven University of Technology, 2002.

${ }^{24} \mathrm{M}$. Fortin, "Old and new finite elements for incompressible flows," Int. J. Numer. Methods Fluids 1, 347 (1981).

${ }^{25}$ C. Cuvelier, A. Segal, and A. A. V. Steenhoven, Finite Element Methods and Navier-Stokes Equations (Reidel, New York, 1986).

${ }^{26}$ A. Segal, Sepran User Manual, Standard Problems and Programmer's Guide (Ingenieursburo SEPRA, Leidschendam, 1984).

${ }^{27}$ Y. Saad, "Sparskit: A basic tool-kit for sparse matrix computations," see http://www.cs.umn.edu/Research/arpa/SPARSKIT/sparskit.html (1994).

${ }^{28}$ One can look at these data in two ways. First of all, we can consider a single type of surfactant, which is present in different concentrations $\Gamma_{r}$. In this case, the interfacial tension coefficient $\sigma_{r}$ will also be different for the droplets, and one has to adjust either the matrix elongation rate $\dot{\epsilon}_{m}$ or the droplet radius $R$ in order to keep the same capillary number. Alternatively, one can consider three different surfactants with different upper concentration limits $\Gamma_{\infty}$, such that they all have the same interfacial tension coefficient $\sigma_{r}$ in the reference state.

${ }^{29}$ Since the calculated concentration $\Gamma$ can temporarily exceed the upper limit of the concentration $\Gamma_{\infty}$ due to numerical errors, we have adapted the equation of state for $\Gamma \leqslant \Gamma_{\infty}$ slightly: we impose a finite interfacial tension $\sigma=\sigma_{r} / 10$ if $\Gamma \geqslant \Gamma_{\infty}$. In reality, $\Gamma_{\infty}$ cannot be exceeded as the Marangoni stresses increase strongly if $\Gamma$ approaches $\Gamma_{\infty}$.

${ }^{30}$ In order to obtain such shapes for clean droplets, different capillary numbers should be used. To obtain the shape correponding to $P e=0$, a relatively low capillary number should be imposed upon the droplet, whereas at higher capillary numbers one would observe shapes similar to the Pe $=100$ case.

${ }^{31}$ Actually, there is also a nonzero concentration in the marker points on the lines connecting the droplets. Since these lines are not physically present, the concentration on these lines are not plotted. The total amount of surfactant associated with these nonphysical lines is less than $1 \%$ of the total amount of surfactant present. 